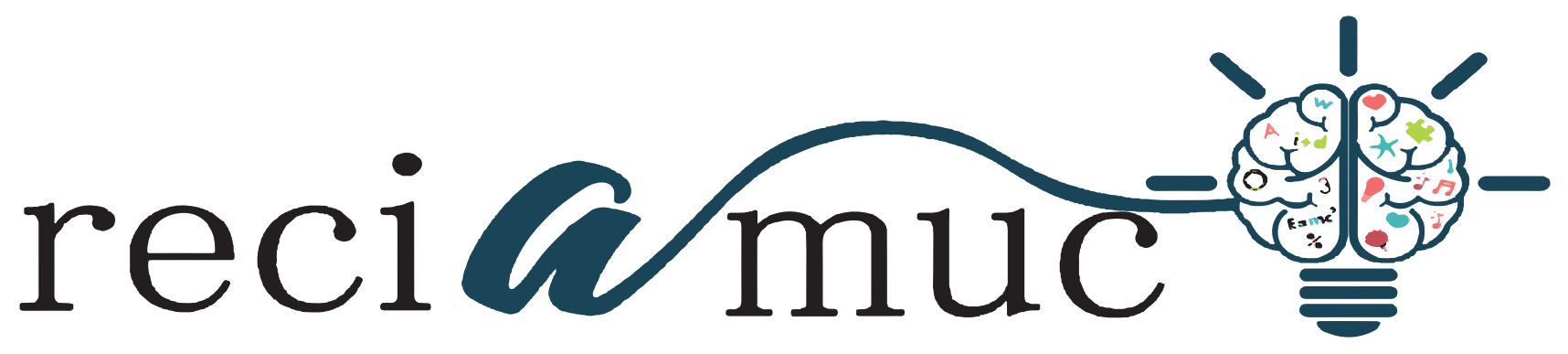

DOI: 10.26820/reciamuc/5.(4).noviembre.2021.13-21

URL: https://reciamuc.com/index.php/RECIAMUC/article/view/717

EDITORIAL: Saberes del Conocimiento

REVISTA: RECIAMUC

ISSN: 2588-0748

TIPO DE INVESTIGACIÓN: Artículo de Revisión

CóDIGO UNESCO: 33 Ciencias Tecnológicas

PAGINAS: $13-21$

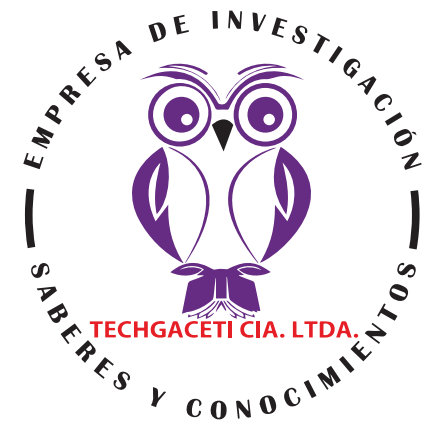

\title{
TIC y las plataformas de concertación bajo condiciones de sostenibilidad
}

ICT and the platforms of agreement under conditions of sustainability TIC e as plataformas de acordo em condições de sustentabilidade

\section{Christian Elías Merizalde Andrade'; Pedro Alfredo Huacon Cruz²; Mario Israel Molina Ortiz ${ }^{3}$}

RECIBIDO: 15/09/2021 ACEPTADO: 05/10/2021 PUBLICADO: 29/11/2021

1. Magister en Sistemas de Información mención en Inteligencia de Negocios; Ingeniero en Sistemas con mención en Telemática; Docente de la Universidad de Guayaquil; Guayaquil, Ecuador; christian.merizalde@ug.edu. ec; (iD https://orcid.org/0000-0002-0982-6752

2. Magister en Administración de Empresas; Contador Público Autorizado; Docente de la Universidad de Guayaquil; Guayaquil, Ecuador; pedro.huacon@ug.edu.ec; iD https://orcid.org/0000-0003-0058-1725

3. Ingeniero en Sistemas Computacionales de la Universidad de Guayaquil; Guayaquil, Ecuador; mario.molinao@ ug.edu.ec; (iD https://orcid.org/0000-0002-5129-098X

\section{CORRESPONDENCIA}

Christian Elías Merizalde Andrade

christian.merizalde@ug.edu.ec

Guayaquil, Ecuador

○ RECIAMUC; Editorial Saberes del Conocimiento, 2021 


\section{RESUMEN}

En la actualidad, existe una creciente preocupación a nivel mundial por el desarrollo sostenible, lo que hace que cada vez más se busquen enfoques endógenos que interactúen globalmente. En este sentido, surgen las plataformas virtuales que buscan ser herramientas de progreso y volver posibles las relaciones de confianza y de colaboración respecto a diversos problemas. Involucran tanto a entidades públicas como privadas que puedan lograr propósitos comunes, ofrecer un espacio neutral para el diálogo político o emprender tareas específicas y, según lo acordado mutuamente, compartir riesgos y responsabilidades, recursos y beneficios. En consecuencia, el presente estudio busca compendiar y referir el uso de las Tecnologías de Información y Comunicación (TIC) para las plataformas de concertación bajo condiciones de sostenibilidad. El tema se desarrollará por medio de los siguientes puntos: la concertación, componente y características; Las TIC y los Objetivos de Desarrollo Sostenible y tecnología sostenible en la gestión y planificación. El modelo de investigación es una revisión de tipo documental bibliográfico. Las TIC son una herramienta de suma importancia para la creación de plataformas virtuales que permitan la concertación o la gestión de múltiples actores para la resolución de problemas a nivel mundial, no obstante, estas aplicaciones tecnológicas deben servir en todo momento para mejorar la vida de las personas de manera fundamental. Estas tecnologías pueden contribuir a reducir la pobreza y el hambre, mejorar la salud, crear nuevos puestos de trabajo, mitigar el cambio climático, mejorar la eficiencia energética y a hacer que las ciudades y las comunidades sean sostenibles. Es imperioso acortar las brechas y las divisiones sociales como consecuencia de la tecnología, con la finalidad de que todos los actores mundiales tengan igualdad de oportunidades.

Palabras clave: Tecnología de Información y Comunicación (TIC), Plataformas, Concertación, Condiciones, Sostenible.

\section{ABSTRACT}

At present, there is a growing concern at a global level for sustainable development, which causes an increasing search for endogenous approaches that interact globally. In this sense, virtual platforms have emerged that seek to be tools for progress and make possible relationships of trust and collaboration regarding various problems. They involve both public and private entities that can achieve common purposes, offer a neutral space for political dialogue or undertake specific tasks and, as mutually agreed, share risks and responsibilities, resources and benefits. Consequently, this study seeks to summarize and refer to the use of Information and Communication Technologies (ICT) for platforms of agreement under conditions of sustainability. The topic will be developed through the following points: the agreement, component and characteristics; ICT and Sustainable Development Goals and sustainable technology in management and planning. The research model is a bibliographic documentary type review. ICTs are a very important tool for the creation of virtual platforms that allow the agreement or management of multiple actors to solve problems worldwide, however, these technological applications must serve at all times to improve the lives of women. people in a fundamental way. These technologies can help reduce poverty and hunger, improve health, create new jobs, mitigate climate change, improve energy efficiency, and make cities and communities sustainable. It is imperative to bridge the gaps and social divisions as a consequence of technology, so that all global players have equal opportunities.

Keywords: Septic shock, intensive care, blood pressure, tissue damage.

\section{RESUMO}

Atualmente, há uma preocupação crescente em nível global com o desenvolvimento sustentável, o que provoca uma busca cada vez maior por abordagens endógenas que interajam globalmente. Nesse sentido, surgiram plataformas virtuais que buscam ser ferramentas para progredir e possibilitar relações de confiança e colaboração em diversos problemas. Envolvem entidades públicas e privadas que podem atingir objetivos comuns, oferecer um espaço neutro para o diálogo político ou realizar tarefas específicas e, conforme mutuamente acordado, compartilhar riscos e responsabilidades, recursos e benefícios. Consequentemente, este estudo procura sintetizar e referir a utilização das Tecnologias de Informação e Comunicação (TIC) para plataformas de pactuação em condições de sustentabilidade. O tema será desenvolvido através dos seguintes pontos: a concordância, componente e características; TIC e Objetivos de Desenvolvimento Sustentável e tecnologia sustentável na gestão e planejamento. O modelo de pesquisa é uma revisão bibliográfica do tipo documental. As TICs são uma ferramenta muito importante para a criação de plataformas virtuais que permitem o entendimento ou gestão de múltiplos atores para a resolução de problemas em todo o mundo, porém, essas aplicações tecnológicas devem servir a todo o momento para melhorar a vida das mulheres. pessoas de uma forma fundamental. Essas tecnologias podem ajudar a reduzir a pobreza e a fome, melhorar a saúde, criar novos empregos, mitigar as mudanças climáticas, melhorar a eficiência energética e tornar as cidades e comunidades sustentáveis. É imperativo preencher as lacunas e divisões sociais decorrentes da tecnologia, para que todos os participantes globais tenham oportunidades iguais.

Palavras-chave: Information and Communication Technology (ICT), Platforms, Agreement, Conditions, Sustainable. 


\section{Introducción}

Desde hace algunas décadas, ha ido aumentando la preocupación por la relación entre la actividad empresarial y el entorno social y ambiental en el que opera. Especialmente en la última década, el interés por la actuación responsable de la empresa ha ido cobrando una relevancia creciente, tanto dentro del ámbito empresarial como fuera del mismo, ya sea por parte de agencias internacionales, ONG, organismos públicos, ámbitos académicos o desde la propia sociedad civil. (García, Guijarro Lomeña, \& Aquije Hernández, 2016, p. 6)

Los avances tecnológicos experimentados durante las últimas décadas del siglo $X X$ $y$ en pleno desarrollo durante el siglo que estamos viviendo, impulsaron una serie de procesos que pueden definirse como una nueva revolución o cambio de paradigma, tanto social, productivo como industrial. La importancia cada vez mayor que adquiere la innovación tecnológica y el conocimiento como factor preponderante en la generación de valor, en un contexto de globalización como el imperante, representan en sí mismos una fuerza transformadora de todos los ámbitos de la actividad social, el trabajo y la producción, el hogar y el consumo, el comercio, el entretenimiento, la socialización y la trasmisión de saberes, la organización de las empresas y los Estados, la provisión de servicios y la circulación de todo tipo de datos y conocimientos. (Mochi, 2012, p. 178)

En este sentido, surgen las plataformas de concertación o plataformas multiactorales de gestión. Según la Real Academia Española, (2020), la acción y efecto de concertar se trata de pactar; asimismo, el concierto se refiere a un ajuste o convenio.

Para Freire Vaccaro \& Rojas Reyes, (2018) una plataforma virtual consiste en un sistema que permite la ejecución de diversas aplicaciones bajo un mismo entorno, ofreciendo a los usuarios la posibilidad de acceder a ellas a través de Internet. Dado esto es evidente la tendencia en que se han vuelto las plataformas digitales. (p. 17)

Basándose en una definición dada por la Asamblea General de la Organización de las Naciones Unidas (ONU):

Las plataformas buscan ser herramientas de progreso y volver posibles las relaciones de confianza y de colaboración respecto a los problemas de tenencia de la tierra entre los diferentes actores. Involucran tanto a entidades públicas como privadas que puedan lograr propósitos comunes, ofrecer un espacio neutral para el diálogo político o emprender tareas específicas y, según lo acordado mutuamente, compartir riesgos y responsabilidades, recursos y beneficios. (Organización de las Naciones Unidas para la Alimentación y la Agricultura - FAO, 2021)

Las plataformas de concertación o multiactores de gestión funcionan como espacios de diálogo y colaboración para el logro de soluciones conjuntas a los problemas en diferentes territorios mundiales, con la participación y en beneficio de múltiples actores.

En este orden de ideas, cabe destacar que, en los últimos años, los procesos de la globalización, la incertidumbre en la estructura y operación de los sistemas de mercado y el impacto negativo que tienen estos sobre el entorno ecológico, han intensificado nuevamente la búsqueda de modelos y estrategias de desarrollo, que se ajusten a la realidad de las demandas específicas de cada localidad, región o país; es decir, enfoques endógenos que interactúan globalmente. (Von Oppeln, 2018, p. 255)

Crespo Coello, (2013) entre los antecedentes de la concertación de alianzas multiactores para entornos sostenibles menciona lo siguiente:

En las décadas de los 70' y 80' surgen progresivamente nuevos paradigmas en las políticas de desarrollo y aparecen los primeros síntomas de la problemática ambiental tanto global como en los niveles nacio- 
nales y locales con afectaciones directas al manejo forestal. Dos procesos más o menos simultáneos son significativos para lo que vendría en el futuro: la planificación participativa para el desarrollo local y, desde la perspectiva del ambiente, la agroforestería social y comunitaria. La década del 90'será muy prolíica en proyectos, nuevas ideas y metodologías con estos enfoques del desarrollo. Y es aquí donde las personas empiezan a ser reconocidas como actores relevantes en la gestión y ya no como simples beneficiarios pasivos del desarrollo, llegándose a una conclusión básica: ninguna intervención de desarrollo puede tener éxito y sentido si se realiza de espaldas a la gente. A modo de una coincidencia feliz, los enfoques de concertación y alianzas se apoyan en la noción de redes en entornos virtuales cada vez más prometedores. Surge así un nuevo concepto de ciudadanía global con una gran capacidad de interacción, superando de esta forma fronteras de espacio y tiempo y permitiendo más espacios de diálogo intercultural. Pero si bien desde la perspectiva de la concertación y de la interacción entre actores, las plataformas virtuales constituyen una oportunidad, por otra parte, la globalización incluye amenazas a la sostenibilidad de los ecosistemas forestales. (p. 2, 3)

En consecuencia, el propósito fundamental del presente estudio es compendiar y referir el uso de las Tecnologías de Información y Comunicación (TIC) para las plataformas de concertación bajo condiciones de sostenibilidad. El tema se desarrollará por medio de los siguientes puntos: la concertación, componente y características; Las TIC y los Objetivos de Desarrollo Sostenible y tecnología sostenible en la gestión y planificación.

\section{Materiales y métodos}

La presente investigación se realizó mediante una metodología de tipo documental o bibliográfica, por cuanto se desarrolló en base a material bibliográfico digital. Este material fue ubicado por medio de equipos tales como ordenadores con conexión a internet.

La ubicación del material requirió del uso buscadores como Google y Google Académico. Asimismo, fue de gran utilidad el portal de la Organización de las Naciones Unidas para la Alimentación y la Agricultura - FAO, Organización de las Naciones Unidas - ONU, Unión Internacional de Telecomunicaciones - ITU, entre otras.

El material del estudio incluyó: informes, protocolos, tesis de grado y otras clases de contenidos. Asimismo, aquellos contenidos repetidos, las editoriales o cartas editoriales, anotaciones académicas y todo documento carente de sustento científico o bajo nivel de evidencia fueron excluidos de la selección.

La investigación fue llevada a cabo durante el mes de septiembre de 2021. Los descriptores para la búsqueda fueron los siguientes: "TIC y plataformas de concertación", "TIC y sostenibilidad"; "Plataformas multiactores y sostenibilidad", "concertación y sostenibilidad". La información fue filtrada bajo los criterios de idioma español. Igualmente, fueron seleccionados aquellos trabajos que tuvieran mayor relevancia y con correlación del tema. Por último, se seleccionaron aquellos trabajos de más actualidad (fecha de publicación: 2006 - 2021).

El equipo investigador manifestó no tener conflicto de intereses.

\section{Resultados}

\section{Concertación}

Compston, (2002) citado por Licha, (2006) define la concertación de la siguiente manera:

Es proceso de toma de decisiones basado en acuerdos entre el Estado y los actores sociales (empresarios y sindicatos principalmente). Su carácter es de co-determinación de las políticas (más allá de la consulta a los actores claves), por lo que se trata de 
un proceso que obliga al gobierno a asumir el compromiso de adoptar políticas públicas específicas inscritas en los acuerdos. Asimismo, la concertación se entiende como un proceso participativo y negociado del Estado con los actores sociales claves del proceso de formación de políticas públicas, a nivel de país o localidad, que afecta la orientación y los contenidos de las mismas, y con importantes implicaciones para toda la sociedad. (p. 5)

\section{Componentes}

Por otra parte, esta autora acerca de los componentes de la concertación, destacan los siguientes:

- Contexto: Se requiere un análisis del entorno para comprender los factores que potencian la necesidad de acuerdos en torno a situaciones particularmente importantes para la comunidad nacional o local donde se ubique la acción de concertación.

- Agenda: La agenda se define en forma participativa, y se va ajustando a lo largo del proceso de diálogo, debate y deliberación ciudadana. Los problemas incluidos en la agenda (y sus posibles soluciones) son el primer resultado del diálogo, y el proceso de concertación gravita en torno a acuerdos sobre acciones prioritarias de desarrollo.

- Actores: El proceso de concertación reconoce e involucra a la pluralidad de actores, y apunta a la articulación de sus distintos intereses, mediante la identificación de áreas de común interés, la complementariedad de distintas capacidades y la búsqueda de coherencia de las acciones de desarrollo. Los actores claves participan en el proceso de concertación, a nivel nacional y subnacional, desde sus respectivas lógicas, intereses y perspectivas. (Licha, 2006, p. 7)

\section{Características de la concertación de ac- tores}

Los actores actúan en el territorio desde su propia lógica e interactúan entre sí; utilizando su capacidad de acción construyen un presente y un futuro, proyectan, comunican, interpretan y actúan de manera intencionada. Generan, a la vez, instituciones que les permiten al mismo tiempo ser sujetos colectivos. En este sentido, las instituciones, producto de la interacción entre los actores, materializan las iniciativas, transformándolas en eficientes y productivas. Ahí radica la importancia de concertar actores frente a propuestas de programas orientados a la generación de capacidades de acuerdo con un proyecto colectivo, que permita diseñar y actuar en políticas territoriales, que no se limiten a resolver problemas de corto plazo, sino que permitan pensar en proyectos de largo alcance. En los distintos niveles: nacional, estatal y local, los actores desarrollan estrategias, ya sea de tipo político (concertación de actores, creación de instituciones, distribución de poder, participación ciudadana, inversiones públicas calificadas, políticas públicas), económico (recursos y atractivos para la localización de empresas, promoción de la economía social, políticas de capacitación y promoción del empleo, políticas para pequeñas y medianas empresas e innovación), cultural (promoción del patrimonio ambiental, histórico, artístico), y científico y tecnológico (inversión en investigación y desarrollo, trasferencias tecnológicas, políticas educativas activas). (Mochi, 2012, p. 183, 184)

\section{Las TIC y los Objetivos de Desarrollo Sostenible}

Desde su creación en el año 2015, la Agenda 2030 para el Desarrollo Sostenible ha proporcionado un modelo para una prosperidad compartida en un mundo sostenible: un mundo en el que todas las personas puedan llevar una vida productiva, vibrante y pacífica en un planeta sano. Durante más de dos años se llevó a cabo un intenso pro- 
ceso de consultas públicas y de interacción con la sociedad civil y otras partes interesadas en todo el mundo, durante el cual se tuvo en cuenta especialmente la opinión de los más pobres y vulnerables, de lo cual resultaron los 17 Objetivos de Desarrollo Sostenible y 169 metas conexas de carácter integrado e indivisible. En base a esta Agenda se han comprometido los líderes del mundo con una acción y un empeño comunes, en camino hacia el desarrollo sostenible del mundo (ver Tabla 1). (Organización de las Naciones Unidas ONU, 2015)

Tabla 1. Objetivos de Desarrollo Sostenible.

\begin{tabular}{|c|c|}
\hline Numero & Objetivo \\
\hline 1 & $\begin{array}{l}\text { Poner fin a la pobreza en todas sus formas y en todo el } \\
\text { mundo. }\end{array}$ \\
\hline 2 & $\begin{array}{l}\text { Poner fin al hambre, lograr la seguridad alimentaria y la } \\
\text { mejora de la nutrición y promover la agricultura } \\
\text { sostenible. }\end{array}$ \\
\hline 3 & $\begin{array}{l}\text { Garantizar una vida sana y promover el bienestar de } \\
\text { todos a todas las edades. }\end{array}$ \\
\hline 4 & $\begin{array}{l}\text { Garantizar una educación inclusiva y equitativa de } \\
\text { calidad y promover oportunidades de aprendizaje } \\
\text { permanente para todos. }\end{array}$ \\
\hline 5 & $\begin{array}{l}\text { Lograr la igualdad de género y empoderar a todas las } \\
\text { mujeres y las niñas. }\end{array}$ \\
\hline 6 & $\begin{array}{l}\text { Garantizar la disponibilidad y la gestión sostenible del } \\
\text { agua y el saneamiento para todos. }\end{array}$ \\
\hline 7 & $\begin{array}{l}\text { Garantizar el acceso a una energía asequible, fiable, } \\
\text { sostenible y moderna para todos. }\end{array}$ \\
\hline 8 & $\begin{array}{l}\text { Promover el crecimiento económico sostenido, inclusivo } \\
\text { y sostenible, el empleo pleno y productivo y el trabajo } \\
\text { decente para todos. }\end{array}$ \\
\hline 9 & $\begin{array}{l}\text { Construir infraestructuras resilientes, promover la } \\
\text { industrialización inclusiva y sostenible y fomentar la } \\
\text { innovación. }\end{array}$ \\
\hline 10 & Reducir la desigualdad en los países y entre ellos. \\
\hline 11 & $\begin{array}{l}\text { Lograr que las ciudades y los asentamientos humanos } \\
\text { sean inclusivos, seguros, resilientes y sostenibles. }\end{array}$ \\
\hline 12 & $\begin{array}{l}\text { Garantizar modalidades de consumo y producción } \\
\text { sostenibles. }\end{array}$ \\
\hline 13 & $\begin{array}{l}\text { Adoptar medidas urgentes para combatir el cambio } \\
\text { climático y sus efectos. }\end{array}$ \\
\hline 14 & $\begin{array}{l}\text { Conservar y utilizar sosteniblemente los océanos, los } \\
\text { mares y los recursos marinos para el desarrollo } \\
\text { sostenible. }\end{array}$ \\
\hline 15 & $\begin{array}{l}\text { Proteger, restablecer y promover el uso sostenible de los } \\
\text { ecosistemas terrestres, gestionar sosteniblemente los } \\
\text { bosques, luchar contra la desertificación, detener e } \\
\text { invertir la degradación de las tierras y detener la pérdida } \\
\text { de biodiversidad. }\end{array}$ \\
\hline 16 & $\begin{array}{l}\text { Promover sociedades pacíficas e inclusivas para el } \\
\text { desarrollo sostenible, facilitar el acceso a la justicia para } \\
\text { todos y construir a todos los niveles instituciones } \\
\text { eficaces e inclusivas que rindan cuentas. }\end{array}$ \\
\hline 17 & $\begin{array}{l}\text { Fortalecer los medios de implementación y revitalizar la } \\
\text { Alianza Mundial para el Desarrollo Sostenible. }\end{array}$ \\
\hline
\end{tabular}


Asimismo, la Organización de las Naciones Unidas, (2015) referida por Ibujés Villacís \& Franco Crespo, (2019), destaca que el apartado 15 de la Agenda 2030 para el Desarrollo Sostenible expresa que "la expansión de las TIC y la interconexión global tienen un gran potencial para superar la brecha digital entre pobres y ricos, y desarrollar sociedades del conocimiento e innovación científica y tecnológica".

En concordancia con este aspecto, la Unión Internacional de Telecomunicaciones (ITU por sus siglas en inglés), (2019) manifiesta que "las tecnologías de la información y la comunicación (TIC) forman la columna vertebral de la economía digital actual y tienen un enorme potencial para avanzar rápidamente en los ODS y mejorar la vida de las personas de manera fundamental".

Las TIC pueden lograr resultados a una escala, velocidad, calidad, precisión y costo que eran inconcebibles hace tan sólo una década. Son medios para suministrar bienes y servicios de calidad en los ámbitos tales como la atención sanitaria, la educación, las finanzas, el comercio, la gobernanza y la agricultura. Pueden contribuir a reducir la pobreza y el hambre, mejorar la salud, crear nuevos puestos de trabajo, mitigar el cambio climático, mejorar la eficiencia energética y a hacer que las ciudades y las comunidades sean sostenibles. Alrededor de la mitad de la población mundial no utiliza Internet. Para cumplir con los 17 ODS es indispensable que la sociedad digital incluya a las poblaciones marginadas, en particular las mujeres y las niñas, los ancianos, las personas con discapacidad, las poblaciones indígenas, los económicamente desfavorecidos, así como los habitantes de países menos adelantados, países en desarrollo sin litoral y pequeños Estados insulares en desarrollo. (Unión Internacional de Telecomunicaciones - ITU, 2018)

La industria de las TIC está cada vez más cuestionada por su impacto negativo en el medio ambiente. Para Naser \& Concha,
(2014) se ha demostrado que esta industria genera altos volúmenes de basura electrónica. Asimismo, el uso de componentes y productos tóxicos en su construcción y los altos consumos de energía eléctrica. En los últimos años, se han visto esfuerzos en la industria de las TIC "que van desde la reducción del uso de productos tóxicos en la fabricación, la adopción de productos reciclables en la fabricación, a campañas de difusión en el uso de baterías y desarrollo de productos más eficientes en el consumo de energía eléctrica". (p. 7. 8)

\section{Tecnología sostenible en la gestión y pla- nificación}

Naser \& Concha, (2014) refieren que es importante formular estrategias tecnológicas sostenibles para la gestión y planificación pública. Uno de los aspectos fundamentales de estas estrategias es la natural tendencia de la tecnología a ser excluyente de los sectores menos alfabetizados digitalmente y que en general son más vulnerables a la denominada "sociedad del conocimiento", en consecuencia, resulta imperioso incorporar elementos que eviten la perpetuación de las brechas y las divisiones sociales como consecuencia de la tecnología. Asimismo, manifiestan:

Estos mecanismos de acceso a los servicios no sólo deben ser accesibles desde un punto de vista económico, sino que también contemplar acciones de una amplia cobertura, adecuada difusión y diversos canales, entre los cuales no debe descartarse totalmente la atención tradicional cara a cara. Una metodología interesante de considerar para la formulación de una estrategia tecnológica sostenible es la técnica del análisis de escenarios y la planificación de escenarios, con ello pueden anticiparse riesgos asociados a la sostenibilidad de políticas públicas de gobierno electrónico en particular su sostenibilidad en el largo plazo. El ejercicio de los escenarios permite la exploración y la evaluación de la vulnerabilidad de las estrategias contra diferentes ambien- 
tes futuros y estadios que alcance el gobierno electrónico. El objetivo, en definitiva, es disponer de una estrategia robusta, esto es, que pueda ajustarse a los diferentes escenarios posibles. (p. 13)

\section{Conclusiones}

Las TIC son una herramienta de suma importancia para la creación de plataformas virtuales que permitan la concertación o la gestión de múltiples actores para la resolución de problemas a nivel mundial, no obstante, debe estas aplicaciones tecnológicas deben servir en todo momento para mejorar la vida de las personas de manera fundamental.

Estas tecnologías pueden contribuir a reducir la pobreza y el hambre, mejorar la salud, crear nuevos puestos de trabajo, mitigar el cambio climático, mejorar la eficiencia energética y a hacer que las ciudades y las comunidades sean sostenibles.

Por último, es imperioso acortar las brechas y las divisiones sociales como consecuencia de la tecnología, con la finalidad de que todos los actores mundiales tengan igualdad de oportunidades.

\section{Bibliografía}

Crespo Coello, P. (2013). La concertación de alianzas multiactores para el Manejo Forestal Sostenible. Programa de Manejo Forestal Sostenible en la Región Andina, Ministerio de Asuntos Exteriores de Finlandia (MAEF). Recuperado el 15 de Septiembre de 2021, de http://repiica.iica.int/docs/ B3394e/B3394e.pdf

Freire Vaccaro, M. R., \& Rojas Reyes, M. E. (2018). Elaboración de una plataforma digital para el desarrollo comercial de las PYMES productoras de miel de abeja del Ecuador para fomentar su consumo. Tesis de pre grado, Universidad Católica de Santiago de Guayaquil, Facultad de Especialidades Empresariales, Guayaquil. Recuperado el 30 de Septiembre de 2021, de http://repositorio.ucsg. edu.ec/bitstream/3317/11498/1/T-UCSG-PREESP-CICE-24.pdf

García, I. H., Guijarro Lomeña, A., \& Aquije Hernández, E. (2016). Huella Social TIC Estudio sobre el impacto en el desarrollo humano de proyectos de tecnologías de la información y Comunicación con participación empresarial en Perú. Madrid: ONGAWA. Recuperado el 12 de Septiembre de 2021, de https://ongawa.org/wp-content/ uploads/2016/06/Huella-social-TIC.pdf

Ibujés Villacís, J. M., \& Franco Crespo, A. A. (2019). Uso de las TIC y relación con los Objetivos de Desarrollo Sostenible en Ecuador. Revista de Ciencias de la Administración y Economía, 9(17), 37-53. Recuperado el 29 de Septiembre de 2021, de https:// www.redalyc.org/journal/5045/504558496003/html/\#redalyc_504558496003_ref19

Licha, I. (2006). Coordinando la Política Social: ¿es la concertación una opción? Serie de Documentos de Trabajo 1-5, Departamento de Integración y Programas Regionales Instituto Interamericano para el Desarrollo Social - INDES, Banco Interamericano de Desarrollo, Washington, D.C . Recuperado el 10 de Septiembre de 2021, de https://publications.iadb.org/publications/spanish/document/ Coordinando-la-Pol\%C3\%ADtica-Social-\%C2\%BFEs-la-Concertaci\%C3\%B3n-una-Opci\%C3\%B3n.pdf

Mochi, P. (2012). Programas para la inclusión digital y la concertación de actores en procesos de desarrollo territorial. POLIS, 8(1), 177-212. Recuperado el 06 de Septiembre de 2021, de http://www.scielo.org.mx/pdf/polis/v8n1/v8n1a7.pdf

Naser, A., \& Concha, G. (2014). Comisión Económica para América Latina y el Caribe - CEPAL. Recuperado el 30 de Septiembre de 2021, de https://repositorio.cepal.org/bitstream/handle/11362/35951/1/ S1420470_es.pdf

Organización de las Naciones Unidas - ONU. (2015). Resolución aprobada por la Asamblea General el 25 de Septiembre de 2015, Transformar nuestro mundo: la Agenda 2030 para el Desarrollo Sostenible. Naciones Unidas. Recuperado el 25 de Septiembre de 2021, de https://www.unfpa.org/ sites/default/files/resource-pdf/Resolution_A_ RES_70_1_SP.pdf

Organización de las Naciones Unidas para la Alimentación y la Agricultura - FAO. (2021). Organización de las Naciones Unidas para la Alimentación y la Agricultura - FAO. Recuperado el 27 de Septiembre de 2021, de https://www.fao.org/ tenure/msps/es/

Real Academia Española. (2020). Real Academia Española. Recuperado el 05 de Septiembre de 2021, de https://dle.rae.es/concertaci\%C3\%B3n

Unión Internacional de Telecomunicaciones - ITU. (2018). Unión Internacional de Telecomunicaciones - ITU. Recuperado el 27 de Septiembre de 
2021, de https://www.itu.int/es/mediacentre/backgrounders/Pages/icts-to-achieve-the-united-nations-sustainable-development-goals.aspx

Unión Internacional de Telecomunicaciones - ITU. (2019). Unión Internacional de Telecomunicaciones - ITU. Recuperado el 28 de Septiembre de 2021, de https://www.itu.int/es/sustainable-world/ Pages/default.aspx
Von Oppeln, C. (2018). Plataformas Multiactorales de Gestión para dinamizar el desarrollo y la innovación agropecuaria local. Estudios del Desarrollo Social: Cuba y América Latina, 13(2), 252-283. Recuperado el 18 de Septiembre de 2021, de http:// scielo.sld.cu/pdf/reds/v6n2/reds13218.pdf

\section{CITAR ESTE ARTICULO:}

Merizalde Andrade, C. E., Huacon Cruz, P. A., \& Molina Ortiz, M. I. (2021).

TIC y las plataformas de concertación bajo condiciones de sostenibilidad.

RECIAMUC, 5(4), 13-21. https://doi.org/10.47464/reciamuc/5.(4).noviem-

bre.2021.13-21

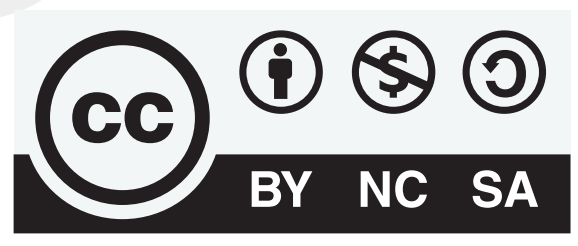

CREATIVE COMMONS RECONOCIMIENTO-NOCOMERCIAL-COMPARTIRIGUAL 4.0. 